\title{
Identification of Human Semen (Spermatozoa) through Different Methods in Forensic Investigation
}

\author{
Harendra Nath Singh ${ }^{1}$, Ranu Paul ${ }^{2}$ \\ ${ }^{1,2}$ State Forensic Science Laboratory, Kolkata, West Bengal (India)-700037 \\ Corresponding Author: Harendra Nath Singh
}

DOI: https://doi.org/10.52403/ijrr.20220314

\begin{abstract}
The presence of seminal stains is very important in crime involving sexual offence. Semen detection is an important factor in forensic investigation to confirm sexual assault in cases of rape. Forensic science laboratory received large numbers of cases involve in sexual offenses for examination and detection of seminal fluid in the exhibits sent by different investigating agencies. A Forensic laboratory receives different types of exhibits for examination of the presence of seminal stains in the exhibits like, garments, cotton swabs, smear in a glass slide, liquid and dry semen, semen mixed with other body fluids, and other articles related to the case. Various methods have been developed in forensic science for identification of semen (sperm). This paper focuses on the basic knowledge of collection seminal stains from the crime scene and detection procedure of human semen (sperm) from the various exhibits in the forensic laboratory. This paper also proposed practical approaches and guideline to help forensic, and law enforcement professionals to deal with the crime scene seminal evidence to avoid contamination, degradation, and loss of the value of the seminal evidence.
\end{abstract}

Keywords: Forensic Science, Spermatozoa, Seminal fluid, Identification of semen, Sexual assaults.

\section{INTRODUCTION}

The identification of semen is frequently of great value in forensic investigation, especially in sexual assault cases (rape, sexual assault, sexual homicide etc). The forensic scientists examine the samples collected from the victims by the medical practitioner or other materials with seminal stains sent by the investigating agencies and link with the suspects according to the findings if any. The scope of a complete identification of semen in a sexual assault case consists of three main functions: First, collection of seminal evidences, Second preservation, and third, identification of seminal stains (sperm) and their interpretation. Many time, due to lack of scientific knowledge of the investigator regarding the proper collection and preservation of the seminal evidence, failure to obtain appropriate results and diminish its value before the court of law. "No biological evidence is resistant to degradation". Thus, careful collection and preservation of biological samples can provide useful information in forensic investigation. Suitable pieces of evidence for identification of semen are limited. Hence, proper precaution for collection and preservation of seminal stains for forensic analysis is required.

\section{Collection of seminal evidence in sexual assault cases}

It is recommended that, before collection of seminal stains on an object, it should be properly documented by either photograph, videography, or sketching. Seminal stains should be air dry properly before packing. Liquid semen should be collected by a clean cotton gauze/cloth by soaking and air drying at room temperature 
properly. Dried or wet seminal stains should be collected by using cotton swab and air drying at room temperature properly before packing. Other articles with seminal stains should be collected as a whole like bed sheets, napkins, pillows, used condoms, garments, etc from the crime scene and air drying at room temperature properly before packing. For sexual assault cases, based upon the condition, necessary samples such as vaginal swab and smear, oral swab, an anal swab, or breast swab should be collected from victims by a qualified medical practitioner and air dry, labeled, and sealed properly. Similarly, the victim's clothes can also be preserved for further examination. In addition, other body fluids such as saliva, urine, blood should be collected in a proper way during the examination.

\section{Avoid contamination of evidence}

Very small amount of semen or seminal stains can be used as evidence. So, in the matter of contamination issues, greater attention is essential when collecting, and preserving the semen or seminal stains for forensic analysis. The integrity of the biological sample is very important in forensic casework. Biological contamination of evidence from another source is a very real possibility, especially when dealing with trace evidence. Following precautions should be taken to avoid contamination of evidence during seminal stains sample collection for forensic analysis.

$>$ Wearing full protective suits, face masks, disposable hand gloves before touching any evidence and change the gloves between handling different items.

$>$ Use disposable instruments or clean them thoroughly before and after handling each sample.

$>$ Avoid touching the area where semen stains may exist.

$>$ Avoid talking, sneezing, and coughing over evidence.
$>$ Avoid touching face, nose, and mouth when collecting and packaging evidence.

$>$ Air-dry the evidence thoroughly before packaging.

$>$ Do not use direct sunlight, hot air blower, or heater to dry.

$>$ Put evidence into new paper bags or envelopes, not into plastic bags. Do not use staples.

$>$ Contact between victim and suspect samples should be avoided at all times.

$>$ Each piece of evidence should be packaged separately into paper bags. Do not use plastic bags.

$>$ Never re-use packaging.

$>$ Do not handle exhibits with bare hands.

$>$ Submit to the laboratory as early as possible without delay.

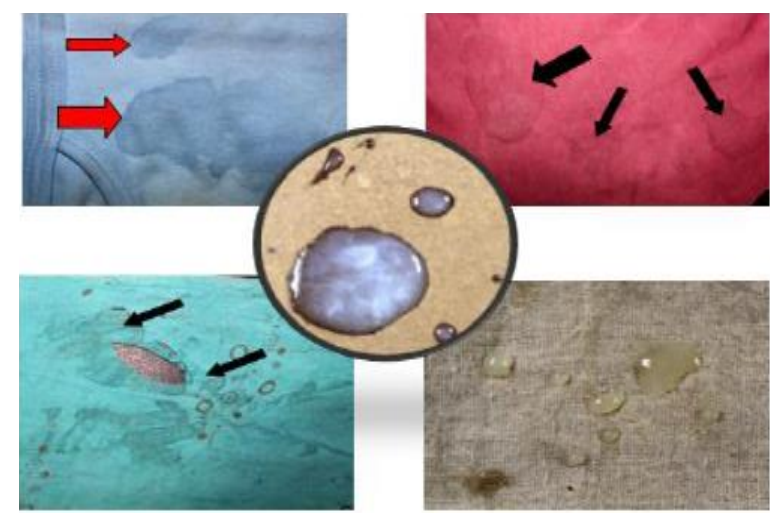

Fig-1: Showing Semen and Seminal stains on cloth.

\section{Biological Characteristics of Seminal Fluids.}

Semen is also known as seminal fluid. It is secreted by the gonads (sexual glands) and other sexual organs of males. The process of discharge of semen is called ejaculation. Semen is also a form of genetic material. The normal healthy male ejaculates approximately 2 to $5 \mathrm{ml}$ of semen that contains seminal fluid and sperm cells (spermatozoa). A normal sperm count ranges from 10000000 to 100000000 spermatozoa per milliliter of semen. Seminal fluid is a complex mixture of glandular secretions and it contains the combined secretions of several accessory glands. The seminal vesicles fluid account for approximately $60 \%$, prostatic fluid secretion approximately $30 \%$, and the 
epididymis and the bulbourethral secretions each approximately $5 \%$ of the ejaculate. Various proteins secreted from the seminal vesicles play a role in the coagulation of the ejaculate. In addition, seminal vesicle fluid contains flavin that causes semen to fluoresce under ultraviolet light. This portion of semen contains high concentrations of acid phosphatase (AP) and prostate-specific antigen (PSA). Both are useful markers for the identification of semen in forensic laboratories(1).

\subsection{Morphology of Spermatozoa.}

The sperm cell can be divided in four parts: The main parts of a spermatozoon are of head, neck, body and thin long tail.

Head: The head is shaped like a flattened ellipse, at the tip of the head is called acrosomal cap and it contains acrosome, a nucleus with densely packed chromosomes and enzymes.

Neck: It contains one typical centriole (the proximal centriole) and one atypical centriole (Distal centriole).

Midpiece: It has a central filamentous core with many Mitochondria spiraled around it. The tail or flagellum is responsible for spermatozoon motility.
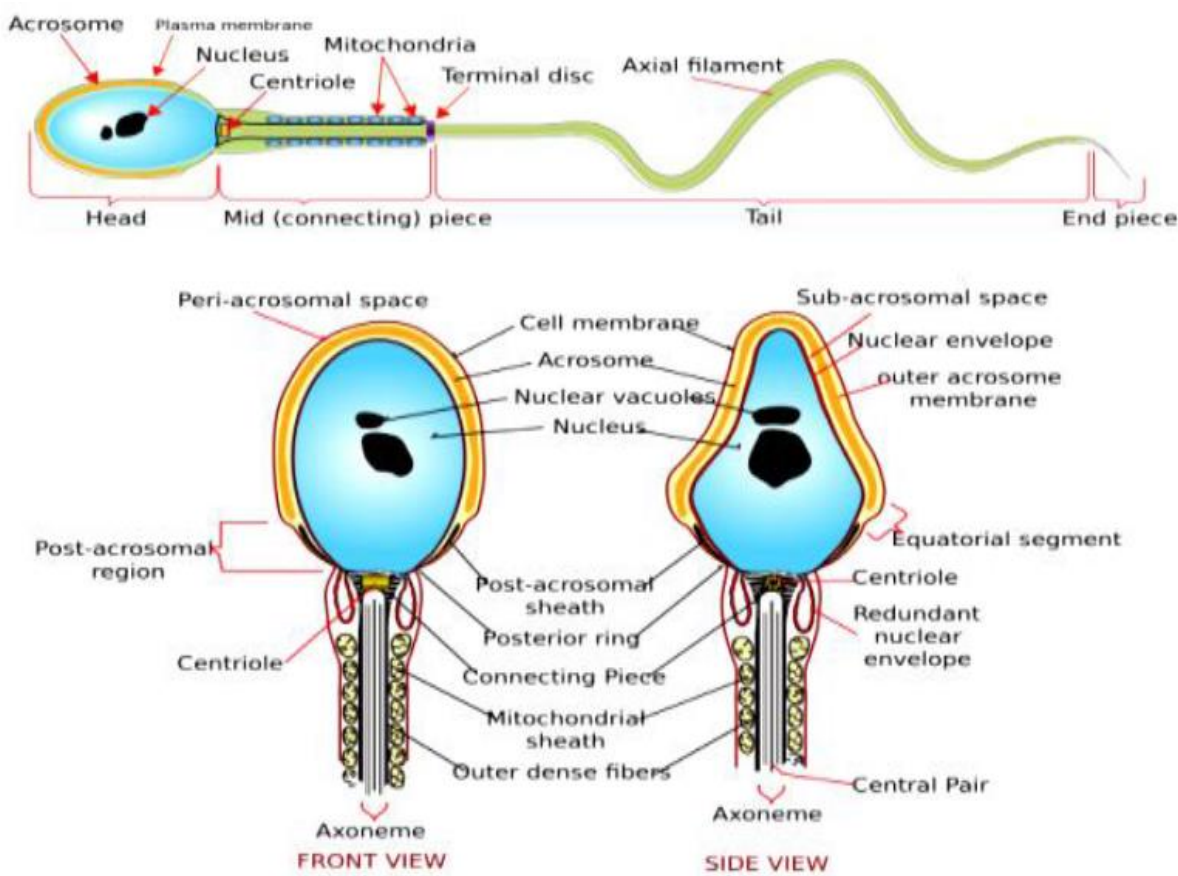

Fig-2: Morphology of Spermatozoa (Source: Wikimedia.org) (2)

\section{Physical examination of Semen:}

Colour: Yellowish white, thick, glossy, opalescent, secretion having a characteristic seminal odour.

Texture: Seminal stains are starchy.

Appearance: Garments sent for forensic examination are usually dirty having variety of stains, in normal light some stains are reddish colour, while others are brown, yellow or faint grey in colour $(3,4)$.

Identification of seminal stains on VSC 6000 instrument.

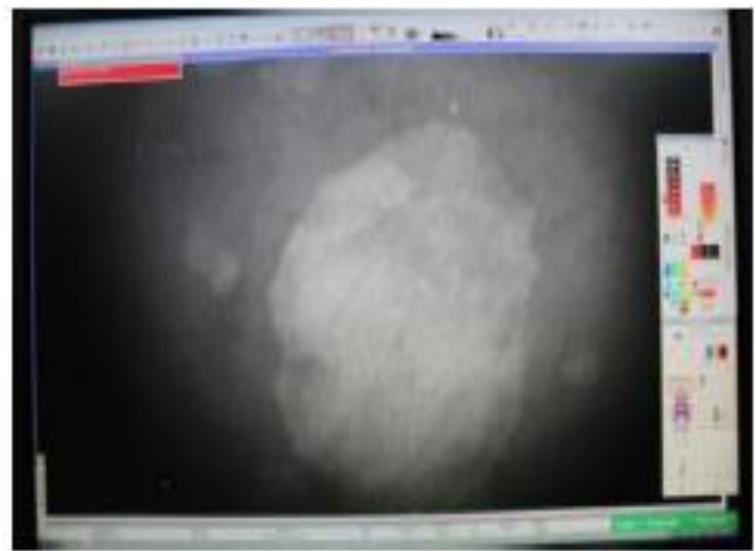

Fig-3: Seminal stains on VSC 6000 instrument using UV light. 


\section{Chemical Methods}

\subsection{Kit used for identification of semen stains.}

Himedia. (HiGenoMB) Semen Identifier Kit. Product Code- ML200-5R

This semen kit has been designed for the detection of human semen from a variety of samples encountered by Forensic laboratories including clothing, bedding, vaginal swabs, and stained surface. This Semen Identifier Kit is based on Acid phosphatase. This test has successfully detected the presence of seminal stains.

\section{Following procedure has been followed for Semen Identification(5).}

1. Thaw the vials containing Reagent SIA and Reagent SIB to room temperature before used.
2. A piece of clean cloth is taken and making it as negative control. A piece of semen stained cloth is taken and making it as positive control. A piece of cloth is taken which is suspected to be stained with semen and making it as test.

3. The vial containing Reagent SIA is opened and added one drop $(50 \mu \mathrm{l})$ each into positive control, Negative control and Test sample.

4. Let the cloth absorb the reagent.

5. The vial containing Reagent SIB is opened and added one drop $(50 \mu 1)$ each into positive control, Negative control and Test sample.

6. A dark purple colour appears on both positive and test samples indicate the presence of semen.

7. Very faint brown or orange colour indicates the negative result.
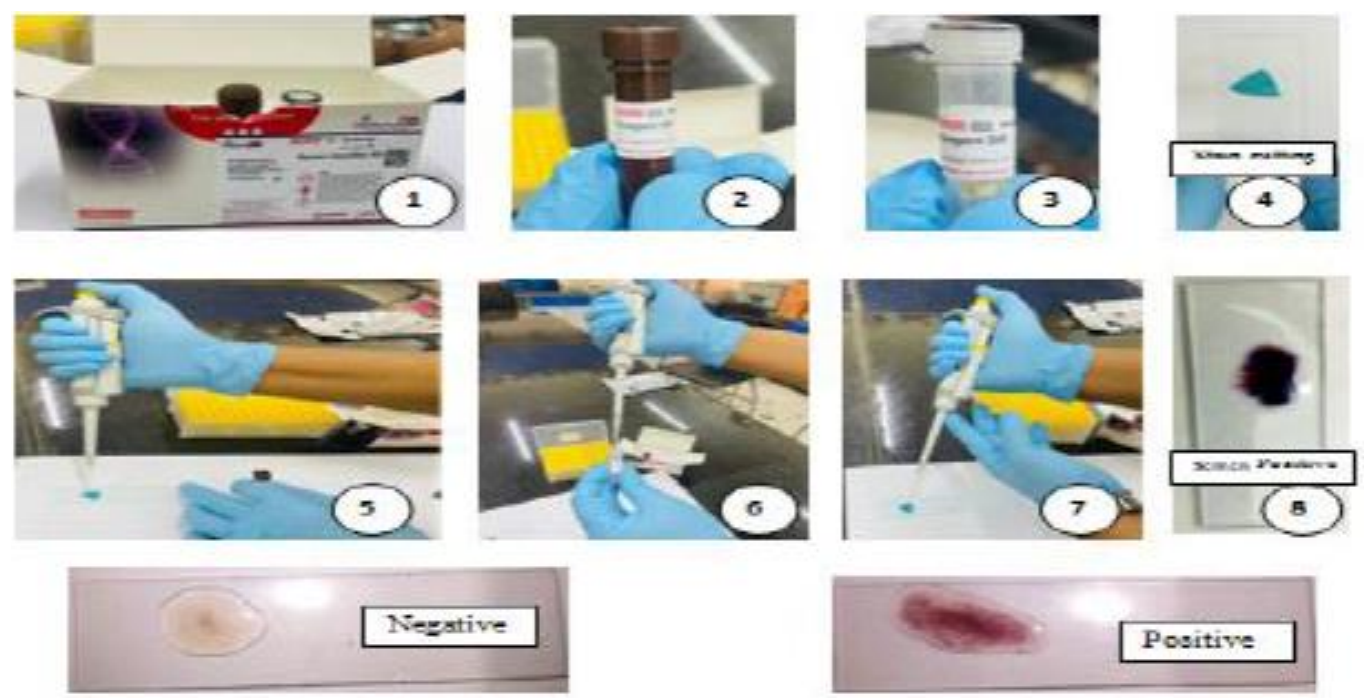

Fig-4: Identification of seminal stain using HiMedia Semen Identifier Kit.

\section{2 $\mathbf{P}^{30}$ Test Using ABA card for Identification of $\operatorname{semen}(6)$. \\ Procedure}

1. Allow the sample to worm to room temperature.

2. Open the pouch and remove the device and dropper.

3. Add 6-7 drops $(200 \mu \mathrm{l})$ of sample to sample well "S" of the test device.

4. Keep 10 minutes for result. Positive results can be seen as early as 1 minute depending upon the $\mathrm{P}^{30}$ concentration.

5. Positive results: If there are two pink line, the test result is positive and indicate that the $\mathrm{P}^{30}$ level is at or above $4 \mathrm{ng} / \mathrm{ml}$.

6. Negative: If there only one pink line, the test result is negative. This may indicate that (a) No $\mathrm{P}^{30}$ is present above $4 \mathrm{ng} / \mathrm{ml}$ in the sample or (b) Presence of high concentration of $\mathrm{P}^{30}$ in the sample (for example undiluted seminal fluid). In such cases the sample may be retested using a 10 to 10,000 fold dilution.

7. Invalid: If there is no pink line visible in the control are of the device the test is inconclusive. 


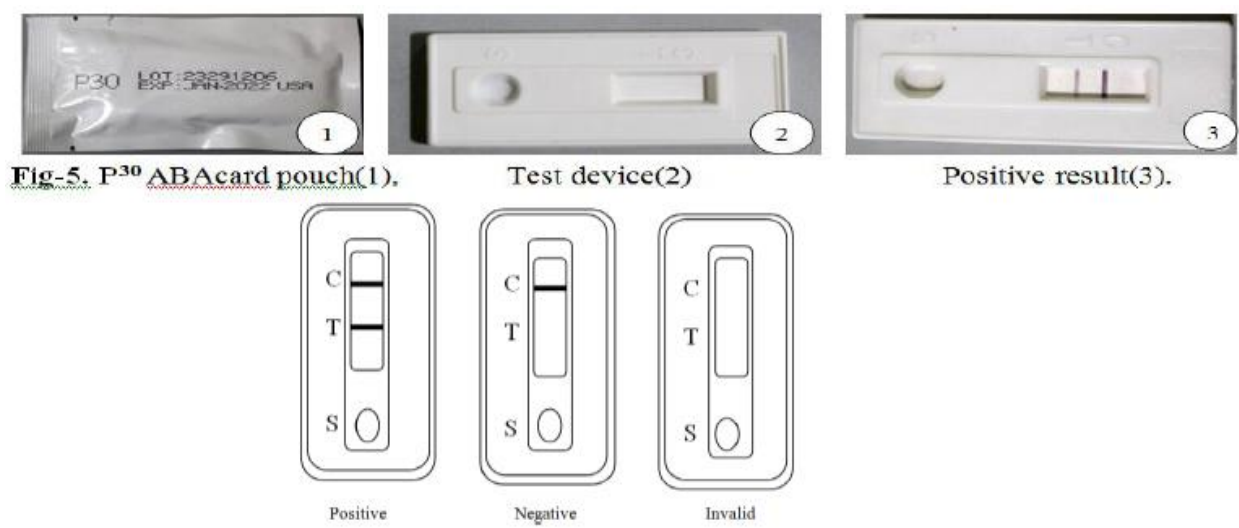

\subsection{Confirmatory Test: Microscopic} Examination of Spermatozoa (3).

Upon obtaining a positive reaction on preliminary test for acid phosphates, the spermatozoa can be extracted from the suspect stain using following procedure:-

\section{Extraction:}

1. A small portion $\left(1 \mathrm{~cm}^{2}\right.$ maximum) of the suspect stain is cut and placed into a test tube.

2. A few drops (as per requirement) of acidulated water is added in the test tube to absorb the stain and kept aside for $1 / 2$ hrs.

3. The stain is then agitated on a vortex or manually, by flicking the tube for freeing the spermatozoa from the stain.

4. When the solution is cloudy, the liquid is withdrawn with a pipette and placed into a centrifuge tube and centrifuged for 30 second.

5. The supernatant is carefully withdrawn and the button of cellular and other insoluble components is collected from the tube and placed on a clean microscopic slide.

6. The slide is either heat-fixed or in dilute $\mathrm{H}_{2} \mathrm{SO}_{4}$ after which the slide is ready for staining.

\section{Staining:}

Method: Gram's staining.

Instrument/equipments used:

1. Glass slide, 2. Hotplate, 3. Centrifuge, 4. Pipette, 5.Water, 6. Gloves, 7. Electric microscope.

8. Scissor, 9. Dropper, 10. Tube and stand.

Chemical used:
Reagent-1: Ammonium oxalate crystal violet solution.

[A: $0.2 \mathrm{~g}$ crystal violate dye $+20 \mathrm{ml}$ of $95 \%$ ethanol]

[B: $0.8 \mathrm{~g}$ Ammonium oxalate $+80 \mathrm{ml}$ distilled water]

[Mix A and B solution to form Reagent-1]

Reagent-2: Gram's Iodine

[1 $\mathrm{g}$ Iodine $+2 \mathrm{~g}$ Potassium Iodine $+300 \mathrm{ml}$ Distilled water]

Reagent-3: Decolorizer. [95\% ethanol] Reagent-4: Safranin Solution.

[Safranin $(2.5 \%$ in $95 \%$ ethanol) $10 \mathrm{ml}+$ 300ml Distilled water]

Following procedure has been followed for preparing the slide.

Step-1. Crystal violet Solution (Reagent-1) is added to the prepared slide and kept for 1 minute.

Step-2. The slide is rinsed with tap water slowly and dried for 1 minute.

Step-3. Gram's Iodine Solution (Reagent-2) is added to the slide and kept for 1 minute.

Step-4. The slide is rinsed with tap water slowly and dried for 1 minute.

Step-5. Decolorizer (Reagent-3) is added and the slide is tilted back and forth to wash away most of the violet colour.

Step-6. The slide is then rinsed with tap water slowly and dried for 1 minute.

Step-7. Safranin Solution (Reagent-4) is added and kept for 1 minute.

Step-8. The slide is rinsed with tap water slowly and air dried.

Step-9. The slide examined under a microscope with oil immersion at $100 \mathrm{X}$ or 1000X. 
Gram positive protein will stain violet and gram negative protein will stain red. Spermatozoa will appear as differentially
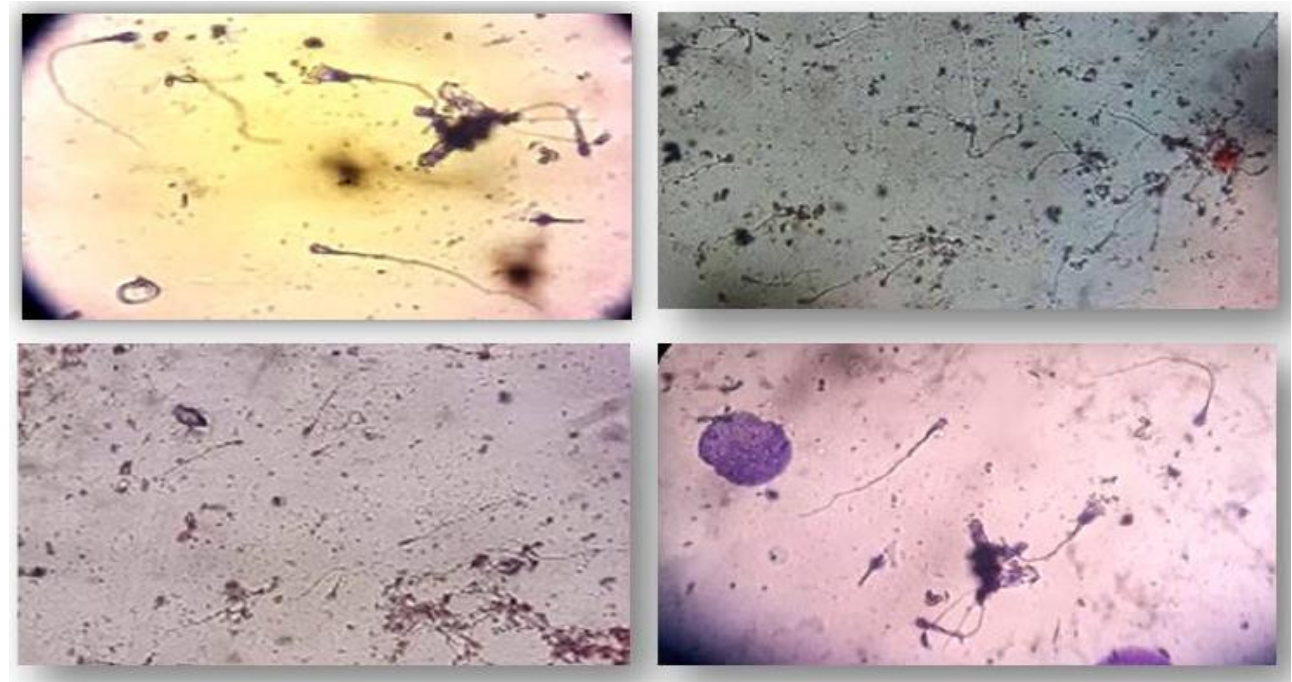

Fig-6: Spermatozoa on microscopic examination.
7. Sample tests and case detection using these methods.

64 positive cases have been successfully detected at different times in forensic lab examination using these three tests i.e. Acid Phosphatase(AP), ProstateSpecific Antigen (PSA, P30) test, and Gram's Staining (microscopic examination/ spermatozoa detection) from various samples such as a vaginal swab, vaginal smear, urethral swab, urethral smear, an anal swab, oral swab, liquid semen, victims clothes, and other materials. Due to various factors (mentioned in point no-8) detection of spermatozoa is difficult from the dried semen sample. The results found that the p30 test (ABA card) gave no false positive but fewer false-negative results were found than the acid phosphatase test. The method P30 is one of the best methods for the identification of seminal stains and the microscopic result for the presence of spermatozoa as the gold standard. In present study, the samples were received from different times and different forms. So, we did not compare the sensitivities test between the AP and p30 test and did not compare the results between these three methods statically. The AP test is the presumptive test for the presence of semen. So, in order to confirm the presence of stained purple bodies with oval-shaped acrosomal caps. semen in the exhibits in forensic examination, a microscopic examination for the presence of spermatozoa (FBS07) or P30 test (FBS06) for the presence of Prostate-Specific Antigen (PSA) must be performed with a positive result of semen.

\section{Reasons for not detecting spermatozoa from the exhibits during forensic examination.}

Detection of one or more sperms is the conclusive proof of the presence of semen. However, it is sometimes difficult to identify sperm from the exhibits of sexual assault cases in the Forensic Laboratory for various reasons, some of which are mentioned here.

i. If the suspect is oligospermic, then his ejaculate may contain only a few sperms. Oligospermia is a male fertility issue characterized by a low sperm count. "The World Health Organization (WHO 2010) classifies sperm counts at or above 15 million sperm per milliliter $(\mathrm{mL})$ of semen as average. Anything below that is considered low and is diagnosed as Oligospermia" (7).

ii. Another reason could be the condition of Aspermia. Aspermia is a condition in which sperms are not produced by the seminiferous tubules of the testes (1). 
Aspermia (dry ejaculate) is the deficiency of semen ejaculation, due to either an inability to transport semen (anejaculation) or to ejaculate in an antegrade direction (retrograde ejaculation) $(8,9)$.

iii. If the suspect has undergone Vasectomy, the sperm cannot enter into the seminal vesicle. "Vasectomy is a surgical procedure for male sterilization or permanent contraception. During the procedure, the male vasa differentia are cut and tied or sealed so as to prevent sperm from entering into the urethra and thereby prevent fertilization of a female through sexual intercourse"(10).

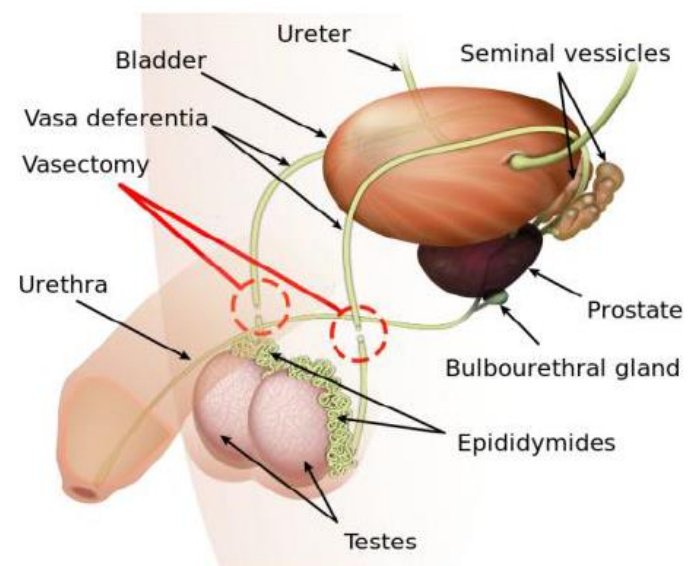

Fig-7: Surgical procedure of Vasectomy (Source: Wikimedia.org) (11)

iv. Use of Condoms.

v. Sperms trapped in the clothing not being extracted into the testing extracts.

vi. Destruction of sperms during handling of the evidence material.

\section{CONCLUSION}

The identification of semen is frequently of great value in forensic investigation, especially in sexual assault cases. The forensic science has various ways of detecting biological evidence left by the offender in sexual assault case. If ejaculation has occurred by the offender in sexual assault case, then the chance of detection semen (spermatozoa) is very high on the exhibits. If the suspect has undergone vasectomy then the sperm may be absent from the semen or seminal stains. In the absence of spermatozoa in the seminal stains on microscopic examinations, others methods can be performed to detect the presence of semen in the exhibits such as fluorescence techniques using UV lights, P30 test and acid phosphatase test. Furthermore, it is important to remember that absence of a positive identification of semen (spermatozoa) on forensic examination does not imply sexual assault has not occurred. Many factors may lead to the absence of semen (spermatozoa) on the exhibits such as use of condom, improper sample collection, sample degradation, amount of semen, physical condition of the offenders etc. The AP test is the presumptive test for the presence of semen. So, in order to confirm the presence of semen in the exhibits in forensic examination, a microscopic examination for the presence of spermatozoa or P30 test for the presence of Prostate-Specific Antigen (PSA) must be performed with a positive result of semen. Using these three tests together is recommended as a forensic tool for detection of semen (spermatozoa)for investigation of sexual assault cases. Hope this paper will help the forensic professional, investigating agencies and judicial personnel to investigate the sexual assault cases.

Acknowledgement: We acknowledge the support from the Government of West Bengal in terms of infrastructures and manpower and Dr. Mousumi Rakshit, Senior Scientific Officer (Biology), RFSL, Jalpaiguri, W.B for her assistance to write this article. We also acknowledge Sri Sanjoy Mukherjee, IPS, Administrator, FSL, West Bengal, in the rank of Director General of Police for all necessary administrative support.

Ethical Standards: All samples used in this study were collected maintaining all legal formalities and the method has been used according to the guidelines mentioned in the Directorate of Forensic Science ServiceIndia. Chemical were collected from HiMedia Laboratory and Abacus 
Diagnostics. This study did not disclose the identity of anybody by any means.

Conflict of Interest: No financial assistance has been received to collect the data or prepare the article.

\section{Source of Funding: None}

\section{REFERENCES}

1. Richard Li: Forensic Biology, CRC Press, Taylor \& Francis Group,2011 PP. 115-130

2. https://upload.wikimedia.org/wikipedia/com mons/9/9f/Complete_diagram_of_a_human _spermatozoa_en.svg

3. Laboratory Procedure Manual, Forensic Biology, Directorate of Forensic Science, Ministry of Home Affairs Govt. of India, New Delhi-2005.

4. https://forensicsdigest.com/semen-as-aforensic-evidence/

5. HiMedia product information (product code, ML200-5R) www.himedialabs.com

6. Abacus Diagnostics, ABAcard $\mathrm{P}^{30}$ Test for the forensic identification of semen, Technical information sheet. Catalog no.308332 www.abacard.com

7. https://www.healthline.com/health/menshealth/oligospermia

8. (2010) The WHO Laboratory Manual for the Examination and Processing of Human Semen ( $5^{\text {th }}$ edn $)$, USA.
9. Mehta A, Sigman M. Management of the dry ejaculate: a systematic review of aspermia and retrograde ejaculation. Fertil Steril. 2015 Nov;104(5):1074-81. doi: 10.1016/j.fertnstert.2015.09.024. Epub 2015 Oct 1. PMID: 26432530

10. https://en.wikipedia.org/wiki/Vasectomy

11. https://commons.wikimedia.org/wiki/File:V asectomy_diagram-en.svg

12. Saferstein, R.: Criminalisties: An introduction of forensic Science, 7th edn.2015. PP. 384-389

13. Kumar,A. Sharma, M: Basics of Human Andrology, Springer Nature Singapore Pte Ltd 2017.

14. Everett Baxte JR:, Complete Crime Investigation Handbook, CRC press 2015.

15. Singh, H.N: Crime Scene Investigation, International Journal of Science and Research (IJSR), 2021 ISSN: 2319-7064, 10(11) PP.642-648.www.ijsr.net

16. Singh, H. N. Collection, preservation and transportation of biological evidence for forensic DNA analysis 2021. www.ijaresm com, 9 (9), pp. 1123-1130.

How to cite this article: Singh HN, Paul R. Identification of human semen (spermatozoa) through different methods in forensic investigation. International Journal of Research and Review. 2022; 9(3): 105-112. DOI: https:// doi.org/10.52403/ijrr.20220314 